\title{
The Distribution of Stellar Populations within Galaxies
}

\author{
Laerte Sodré Jr. and Patricia Martins de Novais \\ Universidade de São Paulo - Instituto de Astronomia, Geofísica e Ciências Atmosféricas - \\ Departamento de Astronomia \\ Rua do Matão, 1226, São Paulo - Brasil \\ email: laerte.sodre@iag.usp.br
}

\begin{abstract}
Stellar populations are fossil records of several physical processes which occur in galaxies and their distribution within these objects may provide important clues on how they form and evolve. By using parameters from image processing we have been developing a new approach to understand the spatial distribution of stellar populations and how this is correlated with the form and evolution of galaxies. In this work we present some results obtained with data from the CALIFA survey.
\end{abstract}

Keywords. methods: data analysis, techniques: image processing, galaxies: evolution, galaxies: formation, galaxies: stellar content, galaxies: structure

\section{Introduction}

Since stellar populations are the results of several processes that galaxies have undergone, the way they are distributed inside galaxies are fingerprints of how these objects form and evolve. Analyzing the spatial distribution of the stellar populations in a pixel by pixel basis is a good way to go further than the usual radial profile studies.

We are developing a new approach to deal with this scientific problem by using tools from image processing. Indeed, we are describing the distribution of stellar populations with 5 sets of parameters: concentration, clumpiness, elongation, compactness and invariant moments. Their regularities for large sample of galaxies are useful to identify patterns that can be associated to the formation processes of these populations within galaxies.

In a pilot project, we have been studying galaxies from the CALIFA survey. Among their science products, this survey has produced many maps of physical properties of galaxies and here we illustrate our procedure with the luminosity weighted stellar age map. Figure 1 shows the distribution of pixels containing populations in 4 different age bins for the galaxy NGC 6125. Clearly, in this case, the older the stars, the more concentrated is the population accordingly with our analysis, as expected in an inside-out galaxy evolution scenario.
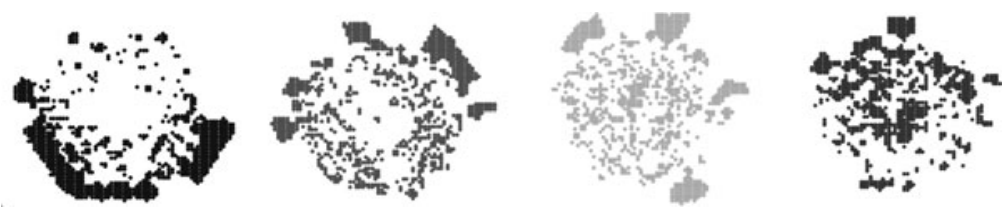

Figure 1. Example of spatially distributed stellar populations, in four age bins, for galaxy NGC6125. From left to right side, the mean age of stellar populations increases. 